\title{
Learning and memory enhancing effects of anthocyanin in black rice extract on cerebral ischaemia in mice
}

\author{
Napapan Kangwan, Komsak Pintha, Watcharaporn Preedapirom, Payungsak Tantipaiboonwong, \\ Orada Chumphukam, Maitree Suttajit
}

School of Medical Sciences, University of Phayao, Phayao 56000 Thailand

*Corresponding author, e-mail: maitree.suttajit@gmail.com

Received 9 Jun 2015

Accepted 18 Oct 2015

\begin{abstract}
The protective effect of anthocyanins extracted from black rice against learning and memory impairment was investigated in mice. In a Morris water maze test, mice with transient cerebral ischaemia required a longer time to find the hidden platform than the sham-operated controls. Daily treatment of cerebral-ischaemia mice with black rice extracts $(125,250$, and $500 \mathrm{mg} / \mathrm{kg}$ body weight) significantly decreased the escape latency and increased the percentage crossing the former platform quadrant in the probe trial. Furthermore, less lipid oxidation was found in the brains of cerebral-ischaemia mice fed with black rice extract. Overall, black rice extract supplementation could be advantageous in the protection against oxidative stress and oxidative damage in the brains of cerebral-ischaemia mice.
\end{abstract}

KEYWORDS: black rice, anthocyanins, lipid peroxidation

\section{INTRODUCTION}

Oxidative stress is characterized by a high production of free radicals that the endogenous antioxidative defence system is unable to counterbalance by. This imbalance condition can cause severe damage to cellular macromolecules. It is widely accepted that increasing oxidative stress level is associated with several age-related degenerative diseases such as neurodegeneration and cognitive impairment ${ }^{1-4}$. In the absence of an effective therapy, brain ageing can increase the susceptibility to Alzheimer's disease, which is the most common cause of dementia $^{5}$. From the report of the World Health Organization, there were nearly 36 million people who suffered from dementia in 2012 and this number will possibly be close to triple in $2050^{6}$.

Although there is not yet an effective therapy to cure dementia, the risk is however preventable. Intensive studies on preventive approaches have been performed to slow down or to delay the onset of diseases ${ }^{7-10}$. Dietary supplementation has been proposed as an effective and safe preventive approach. Daily consumption of coloured vegetables, fruits, and whole grains are not only energy sources but also promote good health due to their high content of biologically active phytochemicals such as polyphenol ${ }^{11-14}$. In plant themselves, phenolic compounds are secondary metabolites mainly produced to protect plants from stresses such as photooxidation, reactive oxygen species, and UV light. Hence, to mimic the plant defensive mechanism, polyphenolic compounds have been used as potent antioxidants that can combat oxidative stress as well as maintain a balance between oxidants and antioxidants.

Rice grains (Oryza sativa) are the major dietary carbohydrate source in Thailand and are the main agricultural product exported to other countries. Pigmented rice, especially black rice, is known for its high antioxidant activity and high phenolic content. Anthocyanins are responsible for the black colour in pericarps. Black rice samples contain anthocyanins $18-26 \%$ of total antioxidant and the major anthocyanin is cyanidin-3-glucoside $(58-95 \%)^{14}$. The positive correlation between a high level of antioxidant activity and a high content of anthocyanin in black rice triggered our interest. These phytochemicals are not only able to reduce oxidative stress but also to modulate cognitive function, especially enhancing memory as well as performing preventive roles in age-related declines of neural function. Thus the health-promoting effect of black rice crude extract was studied. We investigated whether an ethanolic extract from black rice could protect learning and memory impairment in cerebral ischaemia- 


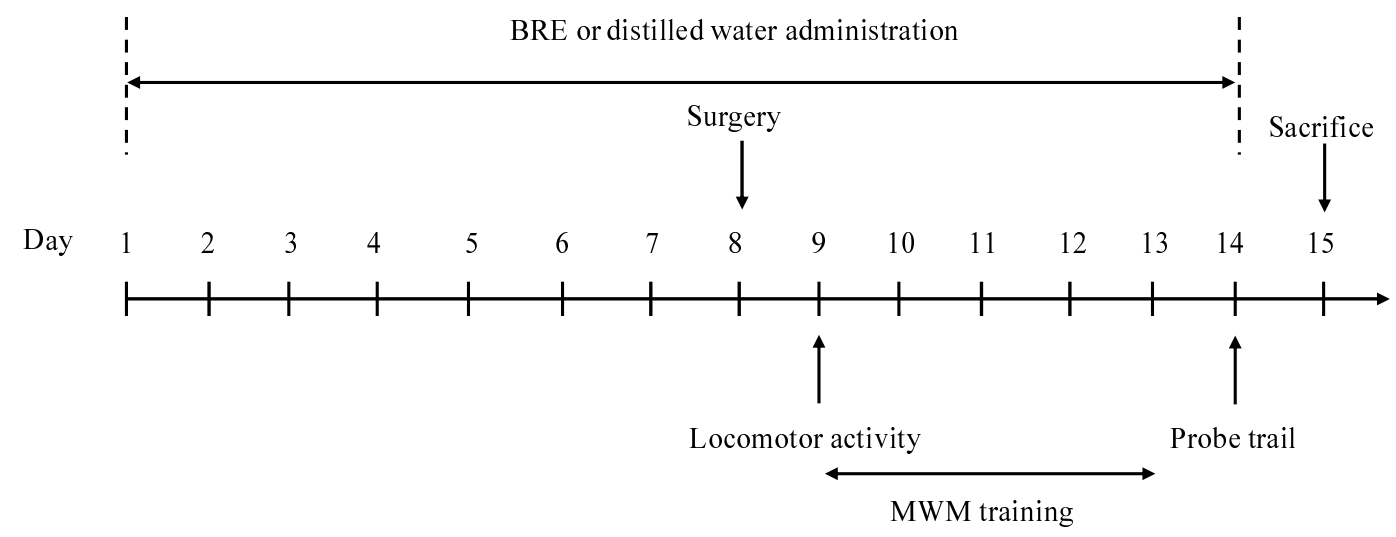

Fig. 1 Experimental design to study the effect of anthocyanin in BRE on cerebral ischaemia in mice model.

induced mice by improving antioxidative status. In addition, to prove that anthocyanins can decrease oxidative damage by lipid peroxidation in brain tissue, the whole brains of mice fed with black rice extract (BRE) were assayed for their malondialdehyde (MDA) levels.

\section{MATERIALS AND METHODS}

\section{Preparation of black rice crude extracts}

Thai black glutinous rice (O. sativa) was obtained from a local market in Phayao province. Dehusked rice grains were ground to a fine powder. Then $250 \mathrm{~g}$ of black rice sample powder was soaked in 11 of $70 \%(v / v)$ ethanol in a shaker $(150 \mathrm{rpm})$ for $12 \mathrm{~h}$ at room temperature. The ethanol extraction was performed twice under the same condition. Subsequently, the extracts were filtered through a Whatman No. 1 filter paper. The filtrates were combined and evaporated using a vacuum rotary evaporator at $70^{\circ} \mathrm{C}$ (Buchi Rotavapor R-200, Flawil, Switzerland). The concentrated aqueous portion was lyophilized into a powder. The dark amorphous powder was then stored at $-20^{\circ} \mathrm{C}$ until use.

\section{Evaluation of phytochemical contents and antioxidant activity}

Total phenolic content (TPC) of the BRE was determined using the Folin-Ciocalteu assay with some modifications as described in Saikia et al ${ }^{15}$. Quantification was expressed as $\mathrm{mg}$ of gallic acid equivalent (GAE) per gram BRE. Total flavonoid content (TFC) was also measured according to Saikia et $\mathrm{al}^{15}$ using aluminium trichloride hexahydrate colorimetric method. The results are expressed as milligram catechin equivalent (CE) per gram sample. The estimation of total anthocyanin content (TAC) was performed using the $\mathrm{pH}$ differential method as described by Sun et $\mathrm{al}^{16}$. The TAC was calculated and expressed as milligrams per litre extract (mg/l). Three replicates were performed for each sample. The composition of BRE was determined using reversed phase high performance liquid chromatography ${ }^{17}$. The free radical scavenging activity of BRE was measured by two methods, using the stable $2,2^{\prime}$ diphenyl-1-picrylhydrazyl radical (DPPH $\left.{ }^{*}\right)$ assay ${ }^{18}$ and reactive oxygen species (ROS) inhibition assay $^{19}$. The results were shown as $50 \%$ of inhibition concentration $\left(\mathrm{IC}_{50}\right)$ value.

\section{Animal grouping, diet, and experiment schedule}

Male ICR mice (4 weeks old, 25-30 g body weight) were obtained from the National Laboratory Animal Centre, Mahidol University (Bangkok, Thailand). Mice were housed for a week under control conditions before initiating the experiments. They were given free access to food and water in control housing conditions including a constant temperature at $25 \pm 2{ }^{\circ} \mathrm{C}$, relative humidity at $60 \pm 10 \%$ and a daily lighting for $12 \mathrm{~h}$. After the acclimation period, the mice were randomly divided into five groups ( $n=8$ per group) including (1) sham-operated control mice, (2) cerebral-ischaemic mice (CI-mice), (3) CI-mice plus BRE treatment at $125 \mathrm{mg} / \mathrm{kg}$ body weight (BW), (4) CI-mice plus BRE treatment at $250 \mathrm{mg} / \mathrm{kg} \mathrm{BW}$, and (5) CI-mice plus BRE treatment at $500 \mathrm{mg} / \mathrm{kg}$ BW. BRE doses were selected as referred to in a previous study ${ }^{20}$. The experiment schedule is shown in Fig. 1. Mice received either distilled water or BRE for 15 days administered orally. The cerebral-ischaemic process was performed on day 8. The Morris water maze (MWM) task was used to test the memory and learning ability of mice in each group from days 9-14 before 
being sacrificed. All experiments were carried out under the approval of the Institutional Animal Ethics Committee, Naresuan University, Thailand.

\section{Transient cerebral ischaemia}

Transient cerebral ischaemia (CI) was induced in mice by bilateral common carotid occlusion as described in $\mathrm{Xu}$ et al ${ }^{11}$, with slight modifications. In brief, mice were anaesthetized by intraperitoneal injection $(40 \mathrm{mg} / \mathrm{kg} \mathrm{BW})$. The bilateral common carotid arteries were exposed and occluded by artery clips for $20 \mathrm{~min}$. Blood was then withdrawn from the cutting off tip of the tail while the arteries were clamped. The cerebral blood flow was then restored by removing the clips and the skin incision was closed. The mice were then kept in a room with the temperature maintained at $25^{\circ} \mathrm{C}$ with a lamp on. Mice in the sham-operated group were subjected to the same process, except that there were no carotid clamping or bleeding.

\section{Locomotor activity and Morris water maze}

To establish whether mice were affected by the cerebral ischaemia, their locomotor activity was tested $24 \mathrm{~h}$ after the operation. A spontaneous movement of each mouse by ambulation and rearing was monitored in an open-field and was recorded for $5 \mathrm{~min}$. After that, spatial learning and memory test was carried out using a Morris water maze (MWM) as described in Morris ${ }^{21}$ and Xu et $\mathrm{al}^{11}$, with modifications. The test was performed in a soundproof room surrounded by various visual cues. The apparatus in the room consisted of a black circular pool $(70 \mathrm{~cm}$ in diameter and $30 \mathrm{~cm}$ in height) divided into four quadrants. The pool was filled with cloudy water (starch solution) to a depth of $23 \mathrm{~cm}$ and the water temperature was maintained at approximately at $25^{\circ} \mathrm{C}$. A $10 \mathrm{~cm}$ diameter platform was fixed at the centre of one quadrant and was hidden $1 \mathrm{~cm}$ below the water surface. The mice were subjected to a three-trial daily training for five consecutive days (days 9-13). The experiment was started by gently placing each mouse in the water facing against the wall of the pool from one of the three starting points in a random manner. The mouse was allowed to swim until it found and climbed onto the platform and the escape latency or time to reach the platform was recorded. If it was unable to reach the platform within $60 \mathrm{~s}$, the trial was stopped and the mouse was placed on the platform. Each mouse was allowed to remain on the platform for $10 \mathrm{~s}$ before being removed from the pool. On day 14 for probe trial, the platform was removed. The mouse was again
Table 1 Phytochemical components in a BRE.

\begin{tabular}{lc}
\hline Phytochemical components $^{\dagger}$ & $\mathrm{mg} / \mathrm{g}$ extract \\
\hline TPC (total phenolic content) & $104.3 \pm 3.4^{*}$ \\
TFC (total flavonoid content) & $21.1 \pm 2.9^{*}$ \\
TAC (total anthocyanin content) & $11.83 \pm 0.61^{*}$ \\
Cyanidin-3-O-glucoside & 4.59 \\
Peonidin-3-O-glucoside & 1.94 \\
\hline
\end{tabular}

The \% yield of BRE was 18.7.

$\dagger$ TPC as gallic acid equivalent; TFC, as catechin equivalent; TAC, as cyanidin-3-O-glucoside equivalent.

Mean \pm SD.

allowed to swim while the crossing time from the former platform quadrant and the crossing time of all quadrants were recorded for $1 \mathrm{~min}$. The ratio of these two crossing times is shown as the percentage of crossing the quadrant of the former platform, which is a representative of spatial memory.

\section{Lipid peroxidation assay in brain homogenates}

On day 15 , the mice were sacrificed by neck dislocation and the brain tissues were collected for lipid peroxidation assay. A lipid peroxidation is related to the formation of a reactive carbonyl product, MDA, which derives from the oxidation of polyunsaturated lipids. To monitor the lipid peroxidation, the level of MDA was measured using thiobarbituric acid reactive substances (TBARS) assay kit (Cayman Chemical Company, USA). The whole mice brain was dissected and homogenized in ice-cold RIPA buffer supplemented with protease inhibitors. The homogenate was centrifuged at $1600 \mathrm{~g}$ for $10 \mathrm{~min}$ at $4^{\circ} \mathrm{C}$. The supernatant was collected to determine the content of MDA.

\section{Statistical analysis}

All data are presented as mean \pm SEM. The statistically significant differences compared between groups were calculated by one-way ANOVA followed by an LSD post-hoc test. The differences were considered significant at the level of $p<0.05$.

\section{RESULTS}

\section{Phytochemical contents and antioxidant activity of ethanolic extract of black rice}

The TPC and TFC were $104.3 \pm 3.4 \mathrm{mg}$ GAE $/ \mathrm{g}$ extract and $21.1 \pm 2.9 \mathrm{mg} \mathrm{CE} / \mathrm{g}$ extract, respectively (Table 1). The TAC, estimated using the $\mathrm{pH}$ differential method, was $11.83 \pm 0.61 \mathrm{mg} / \mathrm{g}$ extract. The two major anthocyanins were found to be cyanidin-3-O-glucoside (predominant) and 


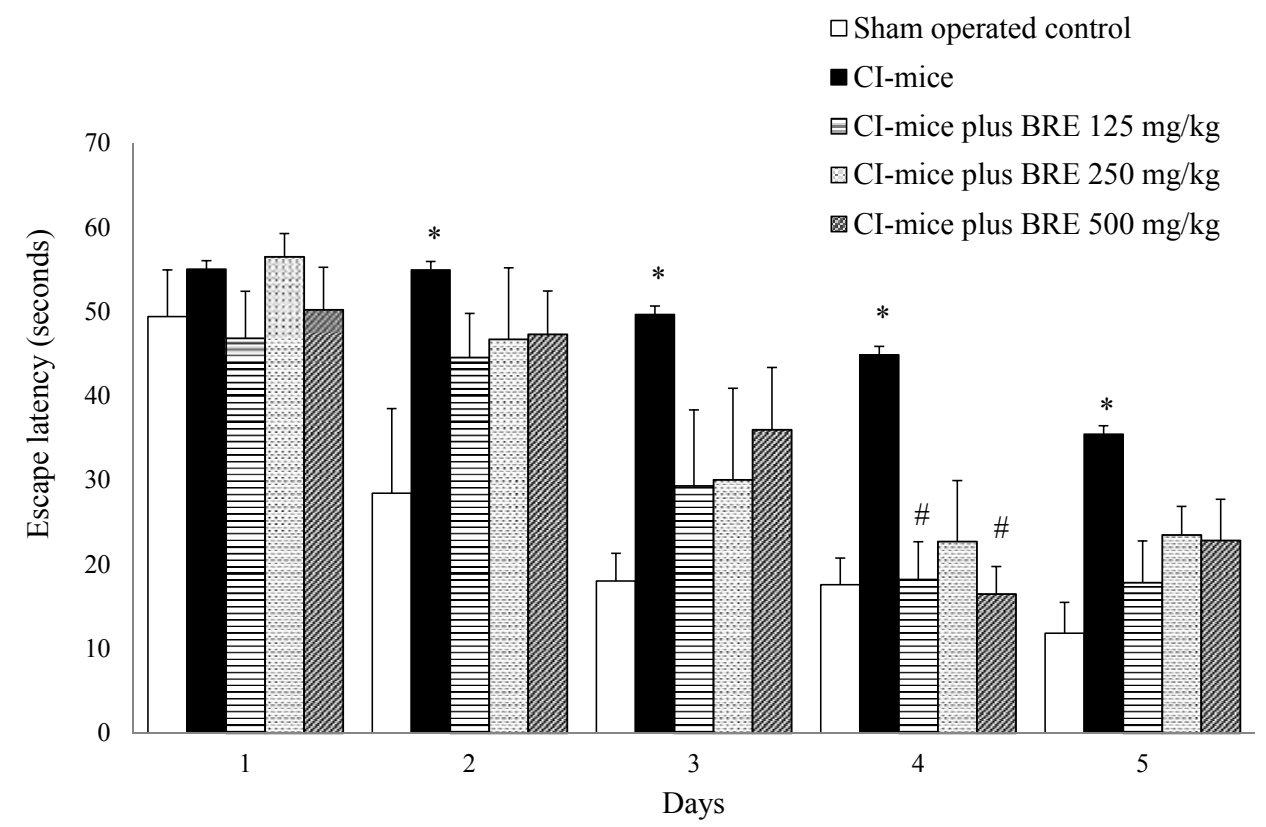

Fig. 2 Effect of BRE on the escape latency in MWM after cerebral ischaemia. Mice were pretreated with distilled water in sham-operated control and in cerebral ischaemia group (CI-mice), and with BRE at doses of 125, 250, and $500 \mathrm{mg} / \mathrm{kg}$ BW once daily for 15 days. The escape latency was evaluated on 5 consecutive days. Data represent the mean of three trials $(n=8)$. * : $p<0.05$ versus sham-operated control; $\#: p<0.05$ versus cerebral ischaemia group (one-way ANOVA-LSD post hoc).

peonidin-3-O-glucoside as shown in an HPLC chromatogram (data not shown). The antioxidant capacity of BRE was evaluated by measuring its DPPH and ROS scavenging activity. The antioxidant effect is proportional to the decolourization of the stable organic radicals. The $\mathrm{IC}_{50}$ value of BRE, measured by the former method, was $45.7 \pm 2.5 \mu \mathrm{g} / \mathrm{ml}$ and for the latter method was $80.2 \pm 3.1 \mu \mathrm{g} / \mathrm{ml}$.

\section{Locomotor activity of mice}

Locomotor activity of mice, $24 \mathrm{~h}$ after the cerebral ischaemia operation, was monitored prior to memory evaluation. The control group was the sham-operated mice. The experimental groups were divided into the CI-mice and CI-mice with BRE daily treatment at different doses. It was found that the cerebral ischaemia did not affect the ambulatory or rearing activity.

\section{Learning and memory assessment}

The learning and memory assessment was performed using MWM test where the mice from each group were given training sessions before a probe trial. The escape latency time was recorded and the average time within the group computed. The data revealed that, during the training trials, the
CI-mice required longer time to find the hidden platform than the sham-operated control group. Interestingly, the group of CI-mice that were supplemented with different doses of BRE $(125,250$, and $500 \mathrm{mg} / \mathrm{kg} \mathrm{BW}$ ) were significantly faster in finding the platform on day 4 of the training trial (Fig. 2). During five days training, the CI-mice plus BRE treatment showed no significantly different performance when compared to the sham-operated control group. To measure the spatial memory, the probe trial was performed on day 14. Two factors were recorded: the time that mice used to cross the quadrant of the former platform and the total time of crossing all quadrants in the water maze. The ratio between these two represents the time that the mice spent searching for the previous platform location and was calculated as the percentage of crossing the quadrant of the former platform (Fig. 3). The percentage of crossing the former platform location increased significantly in the CImice group treated with BRE, especially with BRE at $250 \mathrm{mg} / \mathrm{kg}$ BW.

\section{Lipid peroxidation in brain tissue}

To investigate the level of lipid peroxidation in the brain, the MDA content was determined. It was 


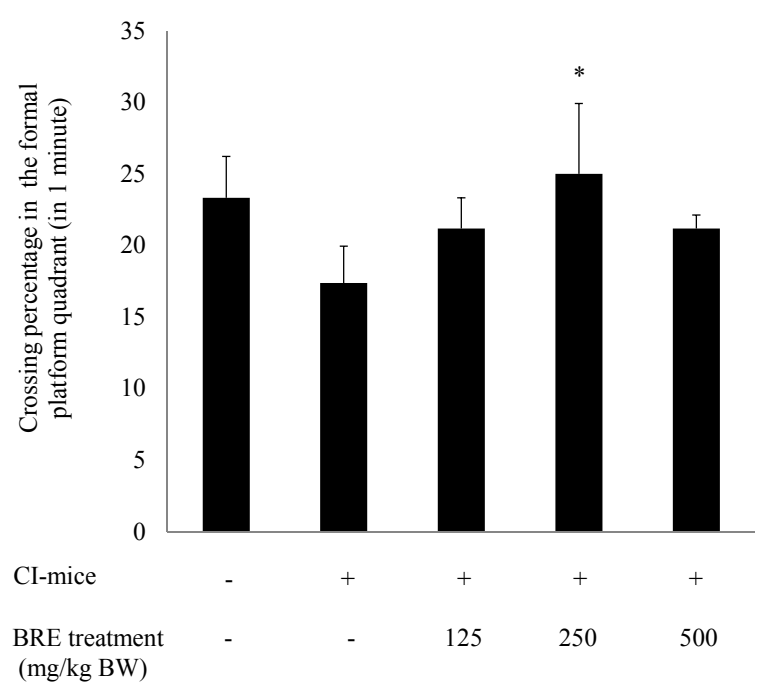

Fig. 3 Effect of BRE on the crossing percentage in the former platform quadrant in probe trial of MWM. Mice were pretreated with distilled water in sham-operated control (CI-mice with negative sign) and in cerebral ischaemia group (CI-mice with positive sign), and with BRE at doses of 125,250 , and $500 \mathrm{mg} / \mathrm{kg} \mathrm{BW}$ once daily for 15 days. The crossing percentage in the former platform quadrant was measured after $24 \mathrm{~h}$ training trail. *: $p<0.05$ versus cerebral ischaemia group (one-way ANOVA-LSD post hoc).

used as a marker of oxidative stress. As shown in Fig. 4, the level of MDA increased in the brain of CI-mice, which was higher than that of the shamoperated control group and the BRE treated group. A significant decrease in the level of MDA was found in the brain of mice treated with high doses of BRE (250 and $500 \mathrm{mg} / \mathrm{kg} \mathrm{BW).} \mathrm{It} \mathrm{implies} \mathrm{that} \mathrm{the} \mathrm{BRE}$ administration was able to alter the concentration of MDA in the brain of mice after cerebral ischaemia.

\section{DISCUSSION}

Numerous studies have shown that various fruits and vegetables contain high amounts of polyphenolic compounds which display high total antioxidant activity. Anthocyanins are responsible for a dark-purple colour and have been reported as potent sources of phytonutrients that exhibit excellent antioxidant capacity ${ }^{20,22-24}$. Pigmented rice or black rice has been reported to have a very high content of total anthocyanins mainly cyanidin-3glucoside and peonidin-3-glucoside ${ }^{14,22-24}$, which corresponds to our investigation. Recent studies show that anthocyanins play an important role in neuroprotection by reducing oxidative stress, pre-

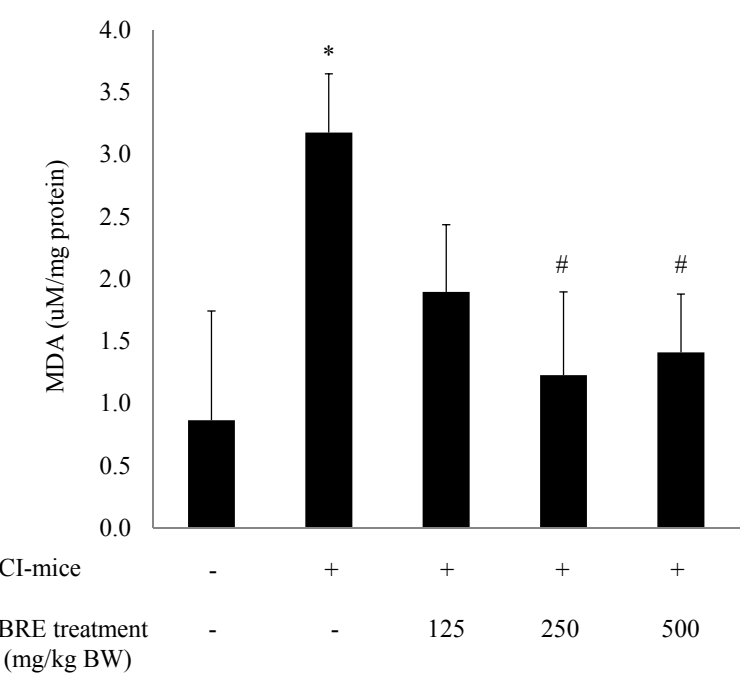

Fig. 4 Effect of BRE on the level of MDA product in brain. Mice were pretreated with distilled water in shamoperated control (CI-mice with negative sign) and in cerebral ischaemia group (CI-mice with positive sign), and with BRE treatment at doses of 125,250 , and $500 \mathrm{mg} / \mathrm{kg}$ BW. Data represent the mean \pm SEM, $(n=8)$. * $: p<0.05$ versus sham-operated control; \#: $p<0.05$ versus cerebral ischaemia group (one-way ANOVA-LSD post hoc).

serving cognitive performance as well as limiting or reversing the deleterious effects of brain ageing. As a daily carbohydrate source for Southeast Asia, we had focused on the investigation of the protective effect of anthocyanin-rich extract from black rice on spatial memory as well as its potential impact against lipid peroxidation in memory deficit mice that have been induced by transient middle cerebral artery occlusion.

Cerebral ischaemia condition results from the blockage of blood vessels and thus decreases the blood supply (as well as oxygen and glucose) to the brain or parts of the brain. It is accompanied by an imbalance of energy metabolism which is promptly followed by the dysfunction of energy dependent ion pumps, depolarization of the neurons, and the generation of reactive oxygen species (ROS). ROS are highly reactive molecules and their accumulation can cause a severe damage such as DNA mutation and lipid and protein dysfunction ${ }^{2-5}$. This condition can cause severe damage to the brain and contributes to numerous neurological illnesses such as Alzheimer's disease and stroke.

A mouse-brain injury model with transient cerebral ischaemia-induced memory deficit is important to the evaluation of the functional consequences of 
cerebral ischaemia. This is also vital to the analysis of the potential therapeutic effect of drugs and food supplements on behavioural testing. Several studies also support the notion that transient cerebral ischaemia could induce learning and memory deficit and neurodegeneration ${ }^{2-4}$. The reason behind this induction is that the damaged brain region was mainly the hippocampus. The hippocampus is a brain area highly associated with spatial learning and memory. This area is also shown to display the most characteristic neuropathological damage in Alzheimer's disease. That is to say, the neuronal injury in the hippocampus may have a positive correlation to the impairment of spatial learning ${ }^{3}$.

In the present study, the normal behaviour of all mice were checked by locomotor activity after surgery. We found that the cerebral ischaemia affected neither general behaviour nor locomotor activity. In addition, the cerebral ischaemia did not affect the swimming ability. Mice were then started training in the MWM after $24 \mathrm{~h}$ surgery (1 day) because we need baseline of MWM on the first day to compare with other days of training. If mice were trained with MWM before surgery, that would not be the baseline study. Furthermore, the results in this study demonstrate that black rice consumption may improve spatial memory in the CI-induced mice using MWM test. The occlusion of bilateral common carotid arteries for 20 min plus hypertension affected neither general behaviour nor locomotor activity $^{11}$. In this study, mice were performed a transient cerebral ischaemia, produced by bilateral common carotid occlusion with removal of $0.3 \mathrm{ml}$ of blood from the tip of the tail. This method produced transient cerebral ischaemia, which affected neither general behaviour nor locomotor activity. However, it produced a deficit in spatial cognition (MWM task). These observations concur with previous reports that transient cerebral ischaemia impairs behavioural performance in a learning and memory tasks in gerbil and mice. It induced however memory deficit as the CI-mice required longer escape latency than the sham-operated control group during the training trials of MWM. The CI-mice that were treated with BRE at 125,250 , and $500 \mathrm{mg} / \mathrm{kg}$ BW significantly shortened the escape latency on day 4 of training trial of MWM (Fig. 2). In addition, the probe trial was tested according to the fact that a mouse with good spatial memory and learning ability should spend much time in the target quadrant where the platform was previously located. The crossing percentage in the previous platform quadrant in the CI-mice was insignificantly lower than the sham-operated control group but significantly higher in the BRE-treatment, especially at $250 \mathrm{mg} / \mathrm{kg}$ BW group during the probe trial of MWM (Fig. 3). This indicates that the supplementation with BRE-anthocyanins resulted in memoryenhancing effects.

Further study on in vivo function of BRE on neuroprotection was demonstrated. As described above, the ROS was induced in brain injury through cerebral ischaemia. The process then induced the generation of lipid peroxides that were degraded to reactive aldehyde products such as MDA. The result of this present study demonstrated that oral intake of BRE slightly inhibited the elevation of MDA levels in CI-mice. Anthocyanin-rich extract has been described for its protective effect against oxidative stress by reducing ROS and increasing antioxidant enzyme activities both in vitro and in vivo. This assumption correlates with the finding that polyphenolic compounds can cross the blood brain barrier. Anthocyanins are delivered and distributed in various brain regions, particularly in the cortex and hippocampus to perform a protection as it is well known that the hippocampus is vulnerable to cerebral ischaemia and related with memory deficit $^{7}$.

To summarize, the anthocyanins in black rice could improve learning and memory as well as reduce the level of lipid peroxidation damage to the brain of CI-mice. Hence black rice may be beneficial for slowing down the memory loss induced or caused by oxidative stress. The protective mechanism of anthocyanins from black rice needs to be studied more intensively. Moreover, further studies on human subjects who are at risk of or at early stage of cognitive problem are required using long-term dietary approach.

Acknowledgements: We are most grateful to the University of Phayao (2010) and Cerebos (Thailand) Corporation Limited (2009) for their kind financial support. The authors thank Assoc. Prof. Dr Boonyong Tantisira for technical assistance.

\section{REFERENCES}

1. Farooqui T, Farooqui AA (2009) Aging: an important factor for the pathogenesis of neurodegenerative diseases. Mech Ageing Dev 130, 203-15.

2. Block F (1999) Global ischemia and behavioural deficits. Progr Neurobiol 58, 279-95.

3. Farkas E, Luiten PG, Bari F (2007) Permanent, bilateral common carotid artery occlusion in the rat: a model for chronic cerebral hypoperfusion-relat- 
ed neurodegenerative diseases. Brain Res Rev 54, 162-80.

4. Pulsinelli WA, Brierley JB, Plum F (1982) Temporal profile of neuronal damage in a model of transient forebrain ischemia. Ann Neurol 11, 491-8.

5. Simão F, Matté A, Matté C, Soares FMS, Wyse ATS, Netto CA, Salbego CG (2011) Resveratrol prevents oxidative stress and inhibition of $\mathrm{Na}^{+} \mathrm{K}^{+}$-ATPase activity induced by transient global cerebral ischemia in rats. $J$ Nutr Biochem 22, 921-8.

6. World Health Organization (2012) Dementia cases set to triple by 2050 but still largely ignored. Available from: www.who.int/mediacentre/news/ releases/2012/dementia_20120411/en/

7. Andres-Lacueva C, Shukitt-Hale B, Galli RL, Jauregui O, Lamuela-Raventos RM, Joseph JA (2005) Anthocyanins in aged blueberry-fed rats are found centrally and may enhance memory. Nutr Neurosci 8, 111-20.

8. Kang TH, Hur JY, Kim HB, Ryu JH, Kim SY (2006) Neuroprotective effects of the cyanidin-3-O- $\beta$-Dglucopyranoside isolated from mulberry fruit against cerebral ischemia. Neurosci Lett 391, 122-6.

9. Shih PH, Chan YC, Liao JW, Wang MF, Yen GC (2010) Antioxidant and cognitive promotion effects of anthocyanin-rich mulberry (Morus atropurpurea L.) on senescence-accelerated mice and prevention of Alzheimer's disease. J Nutr Biochem 21, 598-605.

10. Krikorian R, Shidler MD, Nash TA, Kalt W, VinqvistTymchuk MR, Shukitt-Hale B, Joseph JA (2010) Blueberry supplementation improves memory in older adults. J Agr Food Chem 58, 3996-4000.

11. Xu J, Murakami Y, Matsumoto K, Tohda M, Watanabe H, Zhang S, Yu Q, Shen J (2000) Protective effect of Oren-gedoku-to (Huang-Lian-Jie-Du-Tang) against impairment of learning and memory induced by transient cerebral ischemia in mice. $J$ Ethnopharmacol 73, 405-13.

12. Lee CL, Kuo TF, Wu CL, Wang JJ, Pan TM (2010) Red mold rice promotes neuroprotective sAPPalpha secretion instead of Alzheimer's risk factors and amyloid beta expression in hyperlipidemic $A \beta 40$-infused rats. J Agr Food Chem 58, 2230-8.

13. Spencer JP (2010) The impact of fruit flavonoids on memory and cognition. Br J Nutr 104, S40-7.

14. Laokuldilok T, Shoemaker CF, Jongkaewwattana S, Tulyathan V (2011) Antioxidants and antioxidant activity of several pigmented rice brans. J Agr Food Chem 59, 193-9.

15. Saikia S, Dutta H, Saikia D, Mahanta CL (2012) Quality characterisation and estimation of phytochemicals content and antioxidant capacity of aromatic pigmented and non-pigmented rice varieties. Food Res Int 46, 334-40.

16. Sun J, Yao J, Huang S, Long X, Wang J, García-García E (2009) Antioxidant activity of polyphenol and anthocyanin extracts from fruits of Kadsura coccinea (Lem.) A.C. Smith. Food Chem 117, 276-81.
17. Wongjaikam S, Summart R, Chewonarin T (2014) Apoptosis induction in colon cancer cell lines and alteration of aberrant crypt foci in rat colon by purple rice (Oryza sativa L. var. glutinosa) extracts. Nutr Canc 66, 690-9.

18. Liu D, Shi J, Ibarra AC, Kakuda Y, Xue SJ (2008) The scavenging capacity and synergistic effects of lycopene, vitamin $\mathrm{E}$, vitamin $\mathrm{C}$, and $\beta$-carotene mixtures on the DPPH free radical. LWT Food Sci Tech 41, 1344-9.

19. Saenjum C, Chaiyasut C, Chansakaow S, Suttajit M, Sirithunyalug B (2012) Antioxidant and antiinflammatory activities of gamma-oryzanol rich extracts from Thai purple rice bran. J Med Plant Res 6, 1070-7.

20. Hou Z, Qin P, Ren G (2010) Effect of anthocyaninrich extract from black rice (Oryza sativa L. japonica) on chronically alcohol-induced liver damage in rats. J Agr Food Chem 58, 3191-6.

21. Morris R (1984) Developments of a water-maze procedure for studying spatial learning in the rat. J Neurosci Meth 11, 47-60.

22. Daiponmak W, Senakun C, Siriamornpun S (2014) Antiglycation capacity and antioxidant activities of different pigmented Thai rice. Int J Food Sci Tech 49, 1805-10.

23. Abdel-Aal EM, Young JC, Rabalski I (2006) Anthocyanin composition in black, blue, pink, purple, and red cereal grains. J Agr Food Chem 54, 4696-704.

24. Hu C, Zawistowski J, Ling W, Kitts DD (2003) Black rice (Oryza sativa L. indica) pigmented fraction suppresses both reactive oxygen species and nitric oxide in chemical and biological model systems. J Agr Food Chem 51, 5271-7. 